\title{
Reformulation of Density Functional Theory for $N$-Representable Densities and the Resolution of the $v$-Representability Problem
}

\author{
A. Gonis ${ }^{\mathrm{a}, 1, *}$, X.-G. Zhang ${ }^{\mathrm{b}}$, M. Däne ${ }^{\mathrm{a}}$, G. M. Stocks ${ }^{\mathrm{c}}$, D. M. Nicholson ${ }^{\mathrm{d}}$ \\ ${ }^{a}$ Physical and Life Sciences, Lawrence Livermore National Laboratory, PO Box 808, L-372, Livermore, CA 94551 \\ ${ }^{b}$ Department of Physics and the Quantum Theory Project, University of Florida, Gainesville, FL 32611 \\ ${ }^{c}$ Materials Science and Technology Division, Oak Ridge National Laboratory, Oak Ridge, TN 37831 \\ ${ }^{d}$ Computer Science and Mathematics Division, Oak Ridge National Laboratory, Oak Ridge, TN 37831
}

\begin{abstract}
Density functional theory for the case of general, $N$-representable densities is reformulated in terms of density functional derivatives of expectation values of operators evaluated with wave functions leading to a density, making no reference to the concept of potential. The developments provide proof of existence of a mathematical procedure that determines whether a density is $v$ representable and in the case of an affirmative answer determines the potential (within an additive constant) as a derivative with respect to the density of a constrained search functional. It also establishes the existence of an energy functional of the density that, for $v$-representable densities, assumes its minimum value at the density describing the ground state of an interacting many-particle system. The theorems of Hohenberg and Kohn emerge as special cases of the formalism. Numerical results for one-dimensional non-interacting systems illustrate the the formalism. Some direct formal and practical implications of the present reformulation of DFT are also discussed.
\end{abstract}

Keywords: C. ab initio calculations, D. electronic structure

PACS: 31.10.+z, 31.15.eg, 31.15.vj, 31.50.Df

\section{Introduction}

The theorems of Hohenberg and Kohn [1-3] ushered in density functional theory (DFT), a paradigm in quantum mechanics geared towards the solution of the electronic structure problem defined, for our purposes, as the determination of the quantum states of an interacting system of $N$-electrons in condensed matter. The theorems are developed with respect to the static ground state of an interacting system. The First Theorem shows that the density determines the potential, $v(\mathbf{r})$, acting on an interacting $N$-particle system as a unique (within an additive constant) functional of the density. The Second Theorem establishes the existence of an energy functional of the density,

$$
E_{v}[n]=\int v(\mathbf{r}) n(\mathbf{r d} \mathbf{r})+\left\langle\Psi_{\mathrm{GS}}\left|\hat{T}^{N}+\hat{U}^{N}\right| \Psi_{\mathrm{GS}}\right\rangle
$$

where $\Psi_{\mathrm{GS}}\left(\mathbf{r}_{1}, \ldots, \mathbf{r}_{N}\right)=\Psi_{\mathrm{GS}}\left(\mathbf{r}_{(N)}\right)$ is the wave function of the ground state of an interacting $N$-particle system evolving under the action of an external potential, $v(\mathbf{r})$. The operators, $\hat{T}^{N}$ and $\hat{U}^{N}$, denote, respectively, the kinetic energy and inter-particle interaction of the interacting system. We consider a function of coordinates to be a density if it's everywhere non-negative, normalized to an integer, $N$, and satisfies the so-called kinetic energy condition,

$$
\int\left[\nabla_{\mathbf{r}} \sqrt{n(\mathbf{r})}\right]^{2} \mathrm{~d} \mathbf{r}<\infty
$$

\footnotetext{
${ }^{*}$ Corresponding author

Email address: gonislellnl.gov (A. Gonis)

${ }^{1}$ phone: $+1-925-422-7150$
}

The minimum value of $E_{v}[n]$ occurs at the exact density of the ground state of an interacting system where it equals the energy of the system's ground state.

The expectation value,

$$
\left\langle\Psi_{\mathrm{GS}}\left|\hat{T}^{N}+\hat{U}^{N}\right| \Psi_{\mathrm{GS}}\right\rangle=F_{\mathrm{HK}}[n],
$$

is the Hohenberg and Kohn functional that is determined through knowledge of the density alone being independent of the potential, and is hence referred to as a universal functional of the density.

By construction, the theorems are applicable to densities that are pure-state $v$-representable, i.e., are derived from the solution of a Schrödinger equation corresponding to a given potential (other forms of $v$-representability, such as ensemble $v$ representability can be defined but not considered here). We confine the discussion to pure-state $v$-representability and refer to the condition simply as $v$-rep. As is well known [2, 4], however, the $v$-rep condition introduces a serious difficulty in the theory, known as the $v$-representability problem. Namely, given an arbitrary density the present form of the theory provides no mathematical procedure that can determine whether or not it is $v$-rep.

The main difficulty presented by $F_{\mathrm{HK}}[n]$ springs from the fact that the set of $v$-rep densities is unknown, (given a density it cannot be ascertained as to whether or not it is $v$-rep), and consequently, $F_{\mathrm{HK}}[n]$ is ill-defined [5] (the basic formulation of the $v$-rep problem). An additional difficulty arises because the set of $v$-rep densities is not differentially dense, i.e, there exist den- 
sities that do not come from a potential [6-10] of an interacting (or non-interacting) system. If one ignored these difficulties, the potential would be obtained from the relation,

$$
\frac{\delta F_{\mathrm{HK}}[n]}{\delta n(\mathbf{r})}=-v(\mathbf{r})
$$

modulo an arbitrary constant (that leaves the density unchanged). This relation is generally accepted as to arise from the minimum property of the energy functional, Eq. (1), at the density of the ground state of the system (characterized by $v(\mathbf{r})$ ).

In the conventional interpretation of functional differentiation, the functional derivative, $\frac{\delta F_{\mathrm{HK}}[n]}{\delta n(\mathbf{r})}$, defined through the procedure [2] (where the test function, $\phi(\mathbf{r})$, is arbitrary),

$$
\begin{aligned}
& \lim _{\epsilon \rightarrow 0} \frac{F_{\mathrm{HK}}[n+\epsilon \phi(\mathbf{r})]-F_{\mathrm{HK}}[n]}{\epsilon} \\
= & \int \frac{\delta F_{\mathrm{HK}}[n]}{\delta n(\mathbf{r})} \phi(\mathbf{r}) \mathrm{d} \mathbf{r},
\end{aligned}
$$

at a given density hinges on the evaluation of the Hohenberg and Kohn functional, $F_{\mathrm{HK}}[n+\delta n]$, at densities that differ infinitesimally from $n(\mathbf{r})$. This requires that the density, $n(\mathbf{r})+\delta n(\mathbf{r})$, be $v$-rep, a serious difficulty since the set of $v$-rep densities is not known, as well as requiring the existence of a small dense neighborhood around the density in which the densities are $v$ rep. Assuming such a neighborhood for any density, it can be readily shown that all densities are $v$-rep in contradiction to known facts to the contrary [2, 6, 7]. (A direct proof of the impossibility of differentiating over the domain of $v$-rep densities is given near the end of this paper.)

An additional problem exists in the performance of functional differentiation. Conventional functional differentiation requires that the domain of the functional, say the set of densities, $\{n(\mathbf{r})\}$, with a given normalization, $N$, contains all variations of the form, $n(\mathbf{r}) \rightarrow n(\mathbf{r})+\epsilon \phi(\mathbf{r})$, where $\phi(\mathbf{r})$ is arbitrary (within common caveats of smoothness and integrability), and $\epsilon \rightarrow 0$. It is certainly not true that the set of $v$-representable densities possess this property. Unfortunately, neither does the set of all densities as arbitrary variations may break the requirement of integral normalization.

At least two more difficulties must be mentioned in attempting the performance of the derivative: First, $F_{\mathrm{HK}}[n]$ is defined in terms of an expectation value with respect to the wave function of a pure state (possibly the solution of a Schrödinger equation) and hence with respect to densities with integral normalization, a condition that may fail in the mathematical process of functional differentiation that is based on the use [2] of an arbitrary test function, $\phi(\mathbf{r})$, (such that $\delta n(\mathbf{r})=\epsilon \phi(\mathbf{r})$, with $\epsilon \rightarrow 0$ ). Second, the perceived need to obtain the potential through the functional derivative in (5) requires the knowledge of the value of $F_{\mathrm{HK}}[n]$ at densities other than the one in question, and thus stands in contradiction to the First Theorem [1] specifying that the potential is given through knowledge of the density alone. The requirement that the test function be arbitrary causes further difficulties. A density must be non-negative but for arbitrary functions $\phi(\mathbf{r})$ it is possible that $n(\mathbf{r})+\epsilon \phi(\mathbf{r})<0$. Guarding against the appearance of a negative density compromises the arbitrariness requirement on the test function.
A formal solution to the $v$-representability problem has been sought in terms of potential functional theory [4]. Here, for $N$-particle systems, one introduces functionals of potential, $E_{N, v}[w]=E_{N, v}^{\mathrm{HK}}[n[w]]$, where $E_{N, v}^{\mathrm{HK}}[n[w]]$ is the Hohenberg and Kohn energy functional for $v(\mathbf{r})$, usually denoted by the symbol, $E_{v}[n]$, evaluated at the $v$-rep density, $n(\mathbf{r})$, corresponding to potential, $w(\mathbf{r})$. The stationary points (minima) of $E_{N, v}[w]$ occur at the potentials, $w(\mathbf{r})=v(\mathbf{r})+c$, associated with the minima of the Hohenberg and Kohn functional at the $v$-representable densities corresponding to $v(\mathbf{r})$. The optimized effective potential (OEP) method [11-15] requires this theory as its mathematical justification. Potential functional theory relies on the concept of functionals of potential defining a space that is the dual of that of density functional theory. For such a concept to be valid one must have some way of excluding densities that are not $v$ representable from the space of the densities.

The introduction of the concept of $N$-representability [2, 7, 16], referred to as $N$-rep, namely that all densities can be obtained from antisymmetric, $N$-particle wave functions, established the rigorous foundation of a functional that, when the density is $v$-representable, leads to to $F_{\mathrm{HK}}[n]$. In other words, the set of $v$-representable densities is a subset of all densities, each of which leads to a well-defined functional, $F[n]$. Hence, the set $F_{\mathrm{HK}}[n]$ is a subset of functionals each of which is well defined, so that the subset is well-defined. $N$ representability and the constrained search establish existence but provide no means of determination, identification or construction of $F_{\mathrm{HK}}[n]$. Neither does $N$-representability resolve the difficulties with respect to normalization in the performance of functional differentiation.

The $v$-representability problem would be convincingly solved through the development of a rigorous mathematical procedure that, at the formal level, could determine whether or not the density is $v$-representable. For this to materialize, density functional theory must be formulated entirely based on the density, without reference to a potential. This paper provides a formulation at the same level of conceptual rigor as the constrained search of the existence of such a procedure.

The remainder of the paper takes the following form. The generalization of the Hohenberg and Kohn theorems to general, $N$-representable densities is presented in the following section. Then, we show how the density and corresponding wave function can be determined given the derivative with respect to the density of a functional of the density determined as the minimum expectation value of the sum of the kinetic and interparticle potential operators. A discussion of the formal developments in the paper is given in the final section.

\section{Hohenberg and Kohn Theorems for $N$-representable Densities}

The main result of the paper is a generalization of the Hohenberg and Kohn theorems in terms of general densities without the condition that they are derived from a potential. The generalization is based on the concept of parametric differentiation leading to the determination of rates of change [17] with respect to the density of expectation values of operators in terms 
of wave functions that lead to a density. Now, the arbitrary test function of conventional functional differentiation is replaced by a Dirac delta function leading to the definition,

$$
\frac{\delta F[n]}{\delta n\left(\mathbf{r}^{\prime}\right)}=\lim _{\epsilon \rightarrow 0} \frac{F\left[n(\mathbf{r})+\epsilon \delta\left(\mathbf{r}-\mathbf{r}^{\prime}\right)\right]-F[n(\mathbf{r})]}{\epsilon}
$$

The Dirac delta function is not a proper function. Therefore $n(\mathbf{r})+\epsilon \delta\left(\mathbf{r}-\mathbf{r}^{\prime}\right)$ does not represent a proper charge density expected to yield an energy (expectation) value. Instead, it provides a method of parametric differentiation of any functional of densities through the properties of the delta function using the density $n$ only (and the associated wave functions, if appropriate) [17]. This procedure bypasses the difficulties with conventional functional derivatives when applied to functionals of the density, and allows derivatives with respect to the density of any wave function that leads to the density, and any expectation value of operators with respect to such wave functions.

The formal justification of the derivative is again based on the work of Cioslowski [18-20]. Possibly more important than his demonstration of the explicit construction of any arbitrary subset of the set of wave functions leading to a density, Ciosloski shows that each such wave function possesses a uniquely defined derivative with respect to the density in question. We note that this derivative does not require the determination of functionals (or wave functions) at nearby densities, but is taken at the density under study. This is an important result. Because wave functions leading to a density are differentiable with respect to that density, all expectation values of operators with respect to the density are differentiable, and not only those that lead to a functional. The general expression of the explicit form of that derivative is given in Cioslowski [18-20]-for a summary, see the appendix. A complete formulation is given in [18-20].

Let $\hat{O}=\hat{T}^{N}+\hat{U}^{N}$, and denote the parametric derivative with respect to the density of the expectation value, $\left\langle\Psi\left|\hat{T}^{N}+\hat{U}^{N}\right| \Psi\right\rangle$, with the symbol, $-q_{\Psi}(\mathbf{r})$,

$$
\frac{\delta\left\langle\Psi\left|\hat{T}^{N}+\hat{U}^{N}\right| \Psi\right\rangle}{\delta n(\mathbf{r})}=-q_{\Psi}(\mathbf{r}) .
$$

Conventionally, a constant, $c$, is added to $q_{\Psi}(\mathbf{r})$ to indicate the fact that for $v$-rep densities, the density remains unaffected by a uniform change in the potential. This constant is suppressed in subsequent discussion.

Cioslowski [18] has shown how to construct the set of all antisymmetric, $N$-particle wave functions that lead to a density. Corresponding to each such wave function, construct the quantity,

$$
E_{\Psi}=\int q_{\Psi}(\mathbf{r}) n(\mathbf{r}) \mathrm{d} \mathbf{r}+\left\langle\Psi\left|\hat{T}^{N}+\hat{U}^{N}\right| \Psi\right\rangle,
$$

and, confining derivatives only to first order with respect to the density, note the identity,

$$
\frac{\delta E_{\Psi}}{\delta n(\mathbf{r})}=0
$$

Now, among all $E_{\Psi}$ choose the lowest in value.
In the absence of degeneracy (assumed throughout), the quantity, $E_{\Psi}$, is a minimum if and only if for any wave function, $\Psi^{\prime} \neq \Psi$, that leads to the density, we have,

$$
\left\langle\Psi^{\prime}\left|q_{\Psi}+\hat{T}^{N}+\hat{U}^{N}\right| \Psi^{\prime}\right\rangle>E_{\Psi} .
$$

The existence of a minimum follows from the assumption that the search over the wave functions leading to a density is exhaustive, and that the derivative of any wave function with respect to the density is well-defined $[19,20]$. The term exhaustive is used to describe the characteristic feature of the constrained search as examining all wave functions leading to a density; rather than as a practical implementation of such examination.

We now prove the Generalized First Theorem of Hohenberg and Kohn: The parametric derivative, $q_{\Psi}(\mathbf{r})$, leading to the minimum value of $E_{\Psi}[n]$ is a unique (within an arbitrary additive constant) functional of the density.

In other words, for a given density, there exists only a single minimum among the $E_{\Psi}$ that determines the parametric derivative, $q_{\Psi}(\mathbf{r})$, uniquely within an additive constant.

Consider the functional, $\left\{E_{\Psi}\right\}[n]$, consisting of the set of all $E_{\Psi}$ obtained for a given density, $n(\mathbf{r})$, and consider the minimum value of the set. We show that the minimum leads to a parametric derivative that is a unique (within an additive constant) functional of the density. A schematic representation of this form of the theorem is shown in Fig. 1.

Proof: Consider two antisymmetric wave functions, $\Psi$ and $\Psi^{\prime}$, both leading to $n(\mathbf{r})$ and to the same minimum value of the functional in (8), but with $q_{\Psi}(\mathbf{r}) \neq q_{\Psi^{\prime}}(\mathbf{r})+c$. Because the parametric derivative of the quantity, $\int q_{\Psi}(\mathbf{r}) n(\mathbf{r}) \mathrm{d} \mathbf{r}+\left\langle\Psi^{\prime}\right| \hat{T}^{N}+$ $\hat{U}^{N}\left|\Psi^{\prime}\right\rangle$, with respect to $n(\mathbf{r})$ is non-zero, we have the inequality,

$$
\begin{aligned}
E_{\Psi}[n] & <\left\langle\Psi^{\prime}\left|q_{\Psi}(\mathbf{r})+\hat{T}^{N}+\hat{U}^{N}\right| \Psi^{\prime}\right\rangle \\
& =\left\langle\Psi^{\prime}\left|q_{\Psi^{\prime}}(\mathbf{r})+\hat{T}^{N}+\hat{U}^{N}\right| \Psi^{\prime}\right\rangle \\
& +\int\left[q_{\Psi}(\mathbf{r})-q_{\Psi^{\prime}}(\mathbf{r})\right] n(\mathbf{r}) \mathrm{d} \mathbf{r} \\
& =E_{\Psi}[n]+\int\left[q_{\Psi}(\mathbf{r})-q_{\Psi^{\prime}}(\mathbf{r})\right] n(\mathbf{r}) \mathrm{d} \mathbf{r},
\end{aligned}
$$

since $E_{\Psi}[n]=E_{\Psi^{\prime}}[n]$. Interchanging the roles of the primed and unprimed wave functions, and adding the corresponding expressions, we obtain the contradiction (reductio ad absurdum),

$$
E_{\Psi}[n]+E_{\Psi}[n]<E_{\Psi}[n]+E_{\Psi}[n] .
$$

It follows that there cannot be two different parametric derivatives, $q_{\Psi}(\mathbf{r})$, leading to the same minimum value of the expression in (8), so that $q_{\Psi}(\mathbf{r})$ is a unique functional of the density. As above we can write the functional in (8) in the form, $E_{q[n]}[n]$. For $v$-representable densities, this notation corresponds to the conventional one, $E_{v}[n]$.

The logic of the proof can be summarized as follows: Two minimizing wave functions that give the same density, $n(\mathbf{r})$, lead to different derivatives, $q_{\Psi}(\mathbf{r})$, of the minimized expectation value, $F[n]$, and to a single value of the quantity $E_{\Psi}=$ $\int q_{\psi}(\mathbf{r}) n(\mathbf{r}) \mathrm{d} \mathbf{r}+\left\langle\Psi\left|\hat{T}^{N}+\hat{U}^{N}\right| \Psi\right\rangle$. It is assumed that the two 
derivatives differ by more than a constant. When the roles of the wave functions and the derivatives are interchanged, the derivative of $E_{\Psi}$ fails to vanish implying that each minimum is greater than its common value (itself). The assumption of nondegeneracy allows the use of a strict inequality and the theorem follows.

For $v$-representable densities, the results just established prove that the density of the ground state of a potential determines uniquely the potential (within an additive constant) thus regaining the first of the theorems of Hohenberg and Kohn. However, the theorem emerges as a spacial case for $v$-rep densities of the general result that a density determines uniquely (within an arbitrary constant) the parametric derivative of a functional that is uniquely defined by the density.

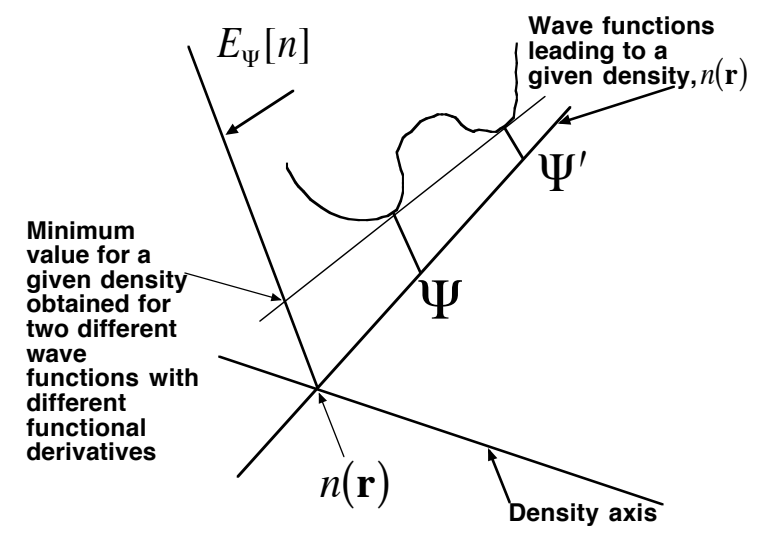

Figure 1: Two wave functions leading to the same density and minimizing $E_{\Psi}[n]$, as discussed in the text.

Generalized Second Theorem: The functional $E_{q[n]}[n]$ assumes its minimum value for the density that leads to $q[n](\mathbf{r})$ where, $\frac{\delta E_{q[n]}[n]}{\delta n(\mathbf{r})}=0$.

Proof: Let there be two wave functions, $\Psi\left(\mathbf{r}_{(N)}\right) \rightarrow n(\mathbf{r})$ and $\Psi^{\prime}\left(\mathbf{r}_{(N)}\right) \rightarrow n^{\prime}(\mathbf{r})$, leading to $q_{\Psi}(\mathbf{r}) \neq q_{\Psi^{\prime}}(\mathbf{r})+c$ such that the parametric derivatives of $E_{q[n]}[n]$ and $E_{q\left[n^{\prime}\right]}\left[n^{\prime}\right]$ at their respective arguments vanish identically. We have,

$$
\begin{array}{r}
E_{q[n]}\left[n^{\prime}\right]=\left\langle\Psi^{\prime}\left|q_{\Psi}+\hat{T}^{N}+\hat{U}^{N}\right| \Psi^{\prime}\right\rangle \\
=\int q_{\Psi}(\mathbf{r}) n^{\prime}(\mathbf{r}) \mathrm{d} \mathbf{r}+\left\langle\Psi^{\prime}\left|\hat{T}^{N}+\hat{U}^{N}\right| \Psi^{\prime}\right\rangle,
\end{array}
$$

so that,

$$
\begin{aligned}
\frac{\delta E_{q[n]}\left[n^{\prime}\right]}{\delta n^{\prime}(\mathbf{r})} & =q_{\Psi}(\mathbf{r})+\frac{\delta\left\langle\Psi^{\prime}\left|\hat{T}^{N}+\hat{U}^{N}\right| \Psi^{\prime}\right\rangle}{\delta n^{\prime}(\mathbf{r})} \\
& =q_{\Psi}(\mathbf{r})-q_{\Psi^{\prime}(\mathbf{r})} \neq 0 .
\end{aligned}
$$

Noting that the non-vanishing of the derivative at a point indicates that the functional at that point is above its minimum completes the proof.

\section{Summary of Density Functional Theory for Ground States}

As emerges from this work, density functional theory is a self-contained body of formalism that allows the determination of a wave function corresponding to an observable, the density of a many-particle interacting system's ground state. The methodology accomplishes this task while avoiding the treatment of the corresponding Schrödinger equation. This direct connection, from classical reality to quantum states describing the outcome of measurement, completes the program of quantum mechanics as a discipline whose fundamental purpose is the determination of states revealed by and explaining the results of measurement.

The independent variable of the theory is the density and its workhorse is the constrained search. Thus, given a density normalized to $N$, the theory prescribes the following sequence of formal steps:

1. Generate the set of all antisymmetric, $N$-particle wave functions that lead to the density.

2. Form the expectation value of the operator, $\hat{T}^{N}+\hat{U}^{N}$, with respect to these wave functions.

3. Identify the wave function, $\Psi_{0}\left(\mathbf{r}_{(N)}\right)$, that gives the lowest expectation value,

$$
F[n]=\left\langle\Psi_{0}\left|\hat{T}^{N}+\hat{U}^{N}\right| \Psi_{0}\right\rangle .
$$

4. Determine the derivative with respect to the density through explicit differentiation of the wave function expanded in the equidensity basis,

$$
\frac{\delta F[n]}{\delta n(\mathbf{r})}=-q(\mathbf{r})
$$

5. and construct the Hamiltonian-like expression,

$$
\hat{H}^{\text {trial }}=-\sum_{i=1}^{N} \frac{1}{2} \nabla_{\mathbf{r}_{i}}^{2}+\sum_{i=1}^{N} q\left(\mathbf{r}_{i}\right)+\frac{1}{2} \sum_{i \neq j} U\left(\mathbf{r}_{i}, \mathbf{r}_{j}\right) .
$$

6. Act on $\Psi_{0}\left(\mathbf{r}_{(N)}\right)$ by $\hat{H}^{\text {trial }}$ and check the satisfaction of the equality,

$$
\hat{H}^{\text {trial }} \Psi_{0}\left(\mathbf{r}_{(N)}\right)=\alpha \Psi_{0}\left(\mathbf{r}_{(N)}\right),
$$

for some real $\alpha$.

7. If the equality fails, the density can be discarded as unphysical, at least with respect to the ground state of a physical system. If it is satisfied, then $q(\mathbf{r})$ is a potential, $\hat{H}^{\text {trial }}$ is the corresponding many-particle Hamiltonian, and $\alpha$ is the eigenvalue of the Hamiltonian's ground state.

Steps 1. to 7. constitute a rigorous mathematical procedure that can decide whether or not a density is $v$-representable thus providing a rigorous solution to the $v$-representability problem. The practical ramifications of the formalism is demonstrated in Section 5

It is seen that the density determines all properties of a system's ground state that may be represented by the density, including the properties of the density itself, in particular whether or not the density is pure-state $v$-representable. 
Clearly, the ability of testing for $v$-representability obviates the need of determining a priori the class of potentials [21] for which the Hohenberg and Kohn theorems may be valid.

The present formulation allows the definition of density functional theory as a body of work in mathematical physics that leads to a solution of the Schrödinger equation for an interacting, many-particle system (that for a ground state of a potential acting on a system) without the explicit solution of the equation. As is to be shown in future work, it also allows the determination of all eigenstates of a Hamiltonian of an interacting system, as well as the determination of states for time-dependent systems. In short, density functional theory is an alternative methodology to that of the Schrödinger equation for determining the complete electronic structure of interacting systems, and is indeed, the only such method currently known. The verification of this statement is provided through other work (currently in preparation) in which excited states and time-dependent systems are treated within density functional theory, through sole knowledge of the density.

\section{Determining the Density}

We now inquire as to whether it is possible to determine the density and the corresponding wave function given the parametric derivative, $q(\mathbf{r})$ (suppressing the notation $q[n]$ in favor of $q$ ).

Consider the functional, where $q(\mathbf{r})$ corresponds to a density, $n(\mathbf{r})$

$$
E_{q}[n]=\int q(\mathbf{r}) n(\mathbf{r}) \mathrm{d} \mathbf{r}+F[n] .
$$

In the absence of degeneracies, let the Slater determinant, $\Phi_{0}\left(\mathbf{r}_{1}, \ldots, \mathbf{r}_{N}\right)$, lead to the same density, $n(\mathbf{r})$, and minimize the expectation value of the kinetic energy of a system of $N$ electrons, establishing the functional,

$$
\begin{aligned}
T_{\text {det }}[n] & =\underbrace{\operatorname{Min}}_{|\Phi\rangle \rightarrow n(\mathbf{r})}\left\langle\Phi\left|\hat{T}^{N}\right| \Phi^{N}\right\rangle \\
& =\left\langle\Phi_{0}\left|\hat{T}^{N}\right| \Phi_{0}\right\rangle .
\end{aligned}
$$

The density is obtained in terms of $\Phi_{0}\left(\mathbf{r}_{(N)}\right)$ by the expression,

$$
n(\mathbf{r})=\sum_{j}\left|f_{j}(\mathbf{r})\right|^{2},
$$

where $f_{j}(\mathbf{r})$ are the single-particle orbitals that enter the construction of the Slater determinant, $\Phi_{0}\left(\mathbf{r}_{(N)}\right)$.

We denote the parametric derivative of $T_{\text {det }}[n]$ with respect to the density by the symbol $q_{\Phi}(\mathbf{r})$, so that

$$
\frac{\delta T_{\mathrm{det}}[n]}{\delta n(\mathbf{r})}=-q_{\Phi}(\mathbf{r})+c .
$$

Now, construct the functional,

$$
F_{s}[n]=\left\langle\Phi_{0}\left|\hat{T}^{N}+\hat{U}^{N}\right| \Phi_{0}\right\rangle,
$$

and note that,

$$
E_{\mathrm{c}}[n]=F[n]-F_{s}[n] \leq 0,
$$

because $\Phi_{0} \neq \Psi_{0}$. Within the present context, the quantity $E_{\mathrm{c}}[n]$ is referred to as the correlation energy. Adding and subtracting $F_{s}[n]$ to the right-hand side of Eq. (19), we have,

$$
E_{q}[n]=\int q(\mathbf{r}) n(\mathbf{r}) \mathrm{d} \mathbf{r}+F_{s}[n]+E_{\mathrm{c}}[n] .
$$

Setting the parametric derivative of the last expression with respect to the density equal to zero, to signify the presence of a minimum, we obtain the following condition (again suppressing arbitrary constants),

$$
\begin{aligned}
q_{\Phi}(\mathbf{r}) & =q_{\Psi}(\mathbf{r})+\frac{\delta\left\{\left\langle\Phi_{0}\left|\hat{U}^{N}\right| \Phi_{0}\right\rangle+E_{\mathrm{c}}[n]\right\}}{\delta n(\mathbf{r})} \\
& =q_{\Psi}(\mathbf{r})+v_{C}(\mathbf{r})+\mu_{c}(\mathbf{r}),
\end{aligned}
$$

where $v_{C}(\mathbf{r})=\frac{\delta\left\langle\Phi_{0}\left|\hat{U}^{N}\right| \Phi_{0}\right\rangle}{\delta n(\mathbf{r})}$ is the Coulomb potential, and $\mu_{c}(\mathbf{r})=$ $\frac{\delta E_{\mathrm{C}}[n]}{\delta n(\mathbf{r})}$ is the correlation potential. Therefore, given the task of obtaining the density and corresponding wave function that lead to the minimum value of the functional in Eq. (19), one searches for the Slater determinant that leads to a parametric derivative satisfying the condition in (26). The implication of these developments to the implementation of DFT based on the Kohn-Sham scheme will be discussed in future work.

\subsection{Connection with Initial Formulations}

For a given density, $n(\mathbf{r})$, implement the constrained search, identify the wave function (quantum state), $\left|\Psi_{0}\right\rangle$, that minimizes the expectation value of $\hat{T}^{N}+\hat{U}^{N}$, construct the derivative, $q_{\Psi_{0}}(\mathbf{r})$, and check whether or not $\left|\Psi_{0}\right\rangle$ is an eigenstate of the operator, $\hat{q}_{\Psi_{0}}+\hat{T}^{N}+\hat{U}^{N}$, i.e., whether or not it satisfies the equality,

$$
\left[\hat{q}_{\Psi_{0}}+\hat{T}^{N}+\hat{U}^{N}\right]\left|\Psi_{0}\right\rangle=\alpha\left|\Psi_{0}\right\rangle
$$

If it does, then the expression above is a Schrödinger equation for an interacting $N$-particle system, $q_{\Psi_{0}}(\mathbf{r})$ is the external scalar potential acting on the system, $\left|\Psi_{0}\right\rangle$ demotes the system's ground state, and $\alpha$ the corresponding ground-state energy. The theorems of Hohenberg and Kohn follow.

With this definition of the potential, we now examine how the conventional functional derivative might be taken for energies that are at minimum. Consider a (parametric) variation of the potential $q_{\Psi_{0}}(\mathbf{r}) \rightarrow q_{\Psi_{0}}(\mathbf{r})+\delta q_{\Psi_{0}}(\mathbf{r})$. The change in the total energy, which is already minimized with respect to all densities, can be evaluated using the Hellmann-Feynman theorem [22],

$$
\delta E_{q}=E_{q+\delta q}-E_{q}=\int \delta q_{\Psi_{0}}(\mathbf{r}) n(\mathbf{r}) d \mathbf{r} .
$$

On the other hand, if the two densities that minimize the total energy for the two potentials are $n(\mathbf{r})$ and $n(\mathbf{r})+\delta n(\mathbf{r})$ (the latter could be obtained from the former using a perturbation expansion in terms of $\left.\delta q_{\Psi_{0}}(\mathbf{r})\right)$, we can also write the change in the total energy as,

$$
\begin{aligned}
\delta E_{q} & =\int \delta q_{\Psi_{0}}(\mathbf{r}) n(\mathbf{r}) d \mathbf{r} \\
& +\int q_{\Psi_{0}}(\mathbf{r}) \delta n(\mathbf{r}) d \mathbf{r}+\int \frac{\delta F[n]}{\delta n(\mathbf{r})} \delta n(\mathbf{r}) d \mathbf{r}
\end{aligned}
$$


These two equations yield,

$$
\int\left\{q_{\Psi_{0}}(\mathbf{r})+\frac{\delta F[n]}{\delta n(\mathbf{r})}\right\} \delta n(\mathbf{r}) d \mathbf{r}=0
$$

If $\delta n(\mathbf{r})$ is an arbitrary function (with the constraint $\int \delta n(\mathbf{r}) d \mathbf{r}=$ $0)$, then the above equation will lead to Eq. (4). However, $\delta n(\mathbf{r})$ can only take a subset of functions, those that correspond to $\delta q_{\Psi_{0}}(\mathbf{r})$. It is therefore possible to find at least one function, $g(\mathbf{r}) \neq 0$, that is perpendicular to all allowed $\delta n(\mathbf{r})$,

$$
\int g(\mathbf{r}) \delta n(\mathbf{r}) d \mathbf{r}=0 .
$$

Thus, we obtain instead,

$$
\frac{\delta F[n]}{\delta n(\mathbf{r})}=-q_{\Psi_{0}}(\mathbf{r})+c g(\mathbf{r})
$$

where $c$ is an arbitrary constant. This proves that it is generally not possible to take functional derivatives with over the domain of only $v$-representable densities. These developments illustrate the principle that (conventional) functional derivatives can be performed only through arbitrary variations of the independent variable, being blocked over domains constrained by conditions such as $v$-representability or normalization.

\section{Numerical Demonstration}

There is an immediate practical implementation of the formalism described above in terms of constructing a test as to whether or not a density is pure-state $v$-representable with respect to a wave function known to leads to the density. We demonstrate with a case of one-dimensional, non-interacting particles confined to a box of length $L$ with zero potential and infinite potential walls.

In this example, the single-particle orbitals are determined through the Schrödinger equation,

$$
\left\{-\frac{1}{2} \nabla_{x}^{2}+v(x)\right\} \phi_{j}(x)=\epsilon_{j} \phi_{j}(x)
$$

and vanish at the edges of the box. Assumed non-degenerate, the single-particle eigenvalues, $\epsilon_{j}$, can be arranged in order of increasing value,

$$
\epsilon_{1}<\epsilon_{2}<\epsilon_{3}<\ldots
$$

where the subscripts, 1,2 , 3, etc., refer to the ground state, first excited state, second excited state, etc., of a single particle under $v(x)$.

When $v(x)=0$ (or a constant), the normalized single-particle wave functions are given by the expressions,

$$
\phi_{n}(x)=\sqrt{\frac{2}{L}} \sin \left(\frac{n \pi x}{L}\right)=A_{n} \sin k_{n} \pi x \quad 0 \leq x \leq L
$$

with quantum numbers $n=1,2,3, \ldots$ In atomic units, the energies take the form,

$$
\epsilon_{n}=\frac{1}{2}\left(\frac{n \pi}{L}\right)^{2}=k_{n}^{2}
$$
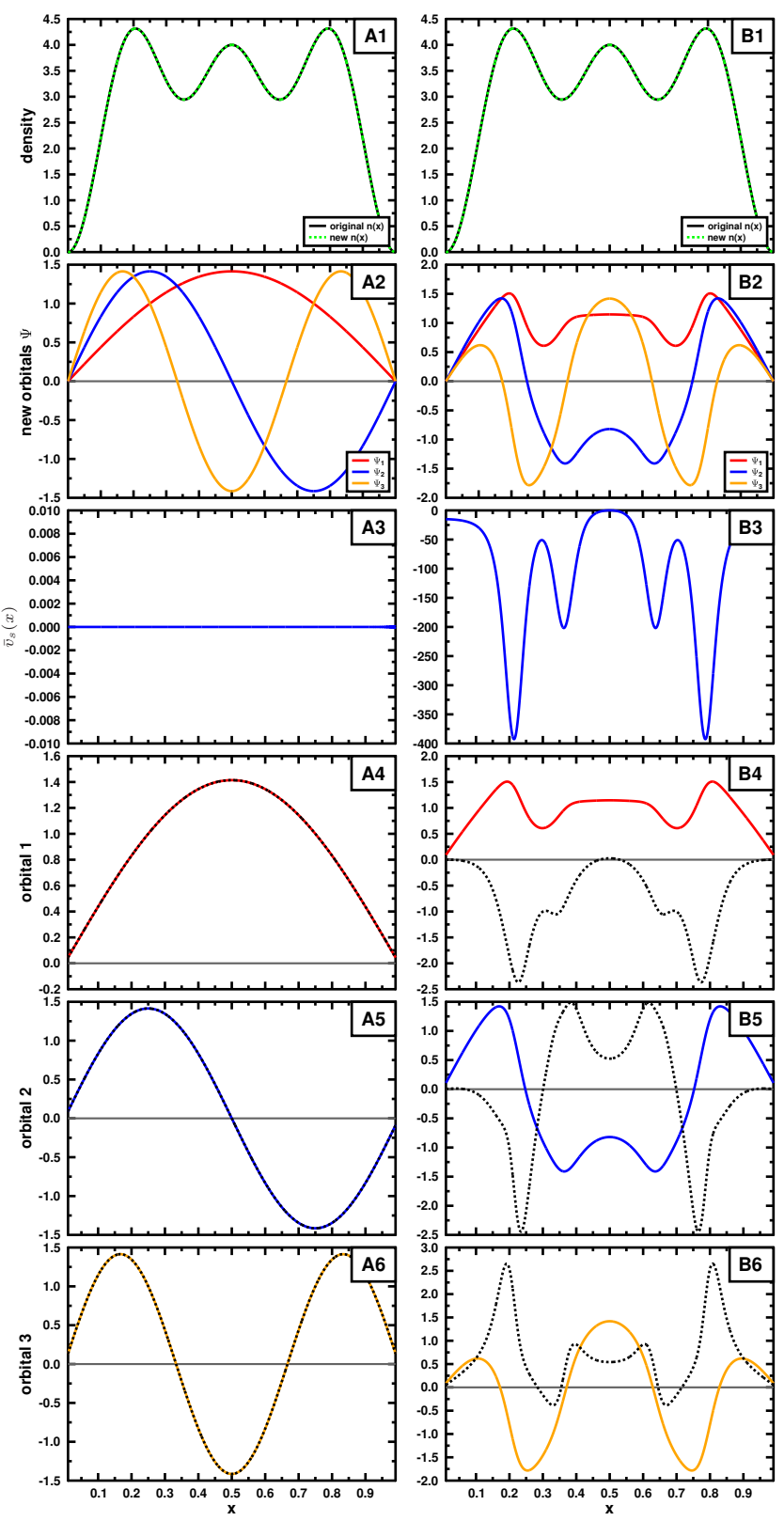

Figure 2: Numerical demonstration of testing for $v$-representability with respect to a system of three non-interacting particles in an one-dimensional box of zero potential and infinite potential walls. In column B, the dotted curves represent the fuctions on the right-hand-side of Eq. (18). 
with the ground-state density for a system of $N$ particles (assumed to be Fermions) given by $n(x)=\sum_{n}\left|\phi_{n}(x)\right|^{2}$.

Figure 2 depicts results obtained with respect to the density, shown in panels A1 and B1, for a system of three particles occupying the three lowest-in-energy orbitals (ground state).

Panel A2 shows the exact orbitals for the ground state of the three-particle system (Eqs. (35)). Constructing the corresponding Slater determinant, $\Phi\left(\mathbf{r}_{1}, \mathbf{r}_{2}, \mathbf{r}_{3}\right)=$ $\operatorname{det}\left|\phi_{1}\left(\mathbf{r}_{1}\right), \phi_{1}\left(\mathbf{r}_{2}\right), \phi_{1}\left(\mathbf{r}_{3}\right)\right|$, taking the expectation value of the kinetic energy operator, $T[n]=\left\langle\Phi\left|T^{3}\right| \Phi\right\rangle$, leads to a derivative, Eq. (16), that is a constant inside the box. Since the derivative determines the potential within an arbitrary constant, the constant is set equal to zero and plotted in panel A3 (the potential inside the box).

Finally, the expression in Eq. (18) is used to test whether the Hamiltonian, $\sum_{j} q\left(x_{j}\right)+\hat{T}^{3}$, applied to each of the orbitals in panel A2 reproduces the orbitals within a constant factor (the corresponding eigen-energy). The application of the Hamiltonian to its eigenstates leads to the eigenstates (multiplied by its eigen-energy) so that the orbitals on the left and right hand-side of Eq. (18) coincide, as shown in panels A4, A5 and A6 for the orbitals, $\phi_{1}, \phi_{2}$ and $\phi_{3}$, respectively.

Panel B3 shows three mutually orthonormal orbitals that lead to the density in panels A1 and B1, but are manifestly different from the exact ground state orbitals of the system (panel A2). Repeating the procedure described with respect to the exact orbitals (i.e., taking the expectation value of the kinetic energy with respect to a determinant constructed from these orbitals, and differentiating with respect to the density the expression resulting from expanding the orbitals in the equidensity basis (see Appendix Appendix B) obtained from the density) leads to a potential inside the box, panel B3, that is clearly not a constant, and also to orbitals obtained on the right-hand-side of Eq. (18) that fail to coincide with input orbitals in panel B2 (and thus do not reproduce the density).

The results of column B also demonstrate an important feature in DFT, emphasized above, namely that not only functionals of the density but also any expectation value with respect to wave functions leading to a density possesses well-defined density derivatives.

Finally, all computational applications based on the present formalism are subject to a numerical tolerance imposed $a$ priori. There may be a number of wave functions that lead to a density and satisfy Eq. (18) within a preset convergence criterion.

\section{Formal and Practical Implications of the Formalism}

In their attempt to prove that an interacting $v$-representable density is also non-interacting $v$-rep, Dreizler and Gross [3] point out that the question is equivalent to the question of the differentiability with respect to the density of the expectation value of the Kohn-Sham kinetic energy functional (expectation value of the kinetic energy with respect to the Kohn-Sham determinant), denoted by $T_{\mathrm{s}}[n]$. They resolve the issue by extending $T_{\mathrm{s}}[n]$ to an ensemble representation, while leaving open the question of the differentiability of the general, constrained search kinetic energy functional, that they denote by $T_{\operatorname{det}}[n]$.

As demonstrated in this paper, all expectation values of the kinetic energy operator with respect to determinants leading to a density, and in particular the kinetic energy functional, are indeed directly differentiable with respect to the density. Thus, $T_{\mathrm{S}}[n]$ as well as $T_{\mathrm{S}}[n]$ are directly differentiable requiring no extension of functionals defined over mixed states.

The test for $v$-representability, Eq. (18) has an important practical ramification in the implementation not only of methods based on DFT, but also on alternative techniques. Consider any methodology, either DFT based of or not, for example a method based on quantum Monte Carlo (because of the generic nature of the comment, we forego references to various QMC techniques) that purports to deliver the ground state energy as well as the ground state wave function (say as a linear superposition of Slater determinants). The test of Eq. (18) can be used to establish the degree of fidelity between the wave function on the left and the right side of (18), thus defining a measure of the accuracy of the wave function determined within the method. Note, for example, that an identity would imply that the wave function is an exact solution of the many-particle Schrödinger equation for the problem at hand.

Likewise, methods that attempt to modify DFT to explicitly include interaction effect, such as LDA+U, self-interaction correction, and schemes using wave functions corrected for electron interaction, can also be easily tested for consistency using the Eq. (18). In particular, the derivative of $T_{\mathrm{s}}[n]$ with respect to the density should yield the negative of the Kohn-Sham potential (modulo a constant), strengthening the argument for the validity of performing derivatives after expansions in the equidensity basis. Of course, satisfying Eq. (18) does not guarantee that a method is correct. But failing this requirement would cast serious doubt on the validity of a method. Thus Eq. (18) provides a guidance on how to winnow down the many possible choices in implementations of the DFT, and, as mentioned above, of methods independent of density functional theory.

\section{Discussion}

For $v$-rep densities, Hohenberg and Kohn's First Theorem establishes a unique functional relationship between the density and the corresponding potential, thus postulating that knowledge of a density, and only knowledge of that density is required in determining the potential. However, Hohenberg and Kohn provide no methodology determining the potential given the density of the potential's ground state. In fact, since the domain of $v$-rep densities is unknown, the Hohenberg and Kohn theorems are ill-founded.

Based on the fact the the set of $N$-representable densities is well-defined (all densities are $N$-representable), the paper provides a formal procedure that allows the determination of whether or not a density describes the ground state of a potential, and in the case of an affirmative answer determines that potential (within an arbitrary constant). Thus, the Hohenberg and Kohn theorems emerge as special cases of a more general formalism that is well defined throughout. 
Finally, in addition to its formal importance, the methodology has direct computational ramifications in the realm of electronic structure calculations, both within as well well as outsde the realm of DFT: In the case of the determination of a groundstate wave function and corresponding ground-state energy, it provides a test of the fidelity and accuracy of the wave function through the consistency implied in Eq. (18). Further investigations of the applicability of the formalism to cases beyond that of the ground state are currently underway.

\section{Acknowledgment}

The work at LLNL is supported by the U.S. DOE under Contract DE-AC52-07NA27344 with LLNS, LLC (AG). Research at ORNL is sponsored by the Division of Materials Sciences and Engineering, Office of Basic Energy Sciences (DMN, GMS), and the Center for Defect Physics in Structural Materials $(C D P)$, an Energy Frontier Research Center funded by the U.S. Department of Energy, Office of Science, Office of Basic Energy Sciences (DMN, GMS, AG). A portion of this research was conducted at the Center for Nanophase Materials Sciences, which is a DOE Office of Science User Facility.

\section{Appendix A. Orbitals and Parametric Derivatives}

Cioslowski [18-20] has provided a formal determination of the set $S_{\Psi \rightarrow} n$ of all antisymmetric $N$-particle wave functions that lead to a density normalized to $N$. This is accomplished by showing that one can construct arbitrarily large subsets of that set, which is logically equivalent to the establishment of the set itself. (At the same time, as also pointed out by Cioslowski [18], any practical implementation of the method will lead to an upper bound to a functional.) A general element of $S_{\Psi \rightarrow n} n$ is written as a linear superpositions of Slater determinants, (in Einstein summation notation),

$$
\Psi\left(\mathbf{r}_{(N)}\right)=C_{P} \Phi_{P}\left(\mathbf{r}_{(N)}\right), \quad C_{P} C^{P}=1,
$$

where $\Phi_{P}\left(\mathbf{r}_{(N)}\right)$ is a Slater determinant of order $N$ constructed from $N$ elements (orbitals), $\psi_{j}(\mathbf{r})$, that form a complete and orthonormal basis in single-particle, three-dimensional coordinate space. The orbitals, $\psi_{j}(\mathbf{r})$, are constructed so that the modulus of the wave function, $\Psi\left(\mathbf{r}_{(N)}\right)$, squared and integrated over all coordinates but one yields the density,

$$
\int\left|\Psi\left(\mathbf{r}_{(N)}\right)\right|^{2} \mathrm{~d} \mathbf{r}_{2} \ldots \mathrm{d} \mathbf{r}_{N}=n\left(\mathbf{r}_{1}\right)
$$

This property follows from the form of the orbitals (see [18]),

$$
\psi_{j}(\mathbf{r})=\frac{\sqrt{n(\mathbf{r})} s_{j}^{u} \phi_{u}(\mathbf{r})}{\sqrt{C^{P} C^{Q} \Xi_{P Q}^{k l} s_{k}^{u} s_{l}^{u} \phi_{u}(\mathbf{r}) \phi_{u}(\mathbf{r})}},
$$

where the functions, $\phi_{u}(\mathbf{r})$, form a complete but not necessarily orthonormal basis in three-dimensional coordinate space, the matrix, $\Xi_{P Q}^{k l}$, is defined by the integral over all coordinates but one of two Slater determinants,

$$
\begin{aligned}
& \int \Phi_{Q}\left(\left(\mathbf{r}_{(N)}\right) \Phi_{P}\left(\mathbf{r}_{(N)}\right) \mathrm{d} \mathbf{r}_{2} \mathrm{~d} \mathbf{r}_{3} \ldots \mathrm{d} \mathbf{r}_{N}\right. \\
&= \int\left\{(-1)^{j+1} \psi_{j}\left(\mathbf{r}_{1}\right) M_{j}^{Q}\left(\mathbf{r}_{2}, \ldots, \mathbf{r}_{N}\right)\right. \\
&\left.\times \quad(-1)^{i+1} \psi_{i}\left(\mathbf{r}_{1}\right) M_{i}^{P}\left(\mathbf{r}_{2}, \ldots, \mathbf{r}_{N}\right)\right\} \mathrm{d} \mathbf{r}_{2} \mathrm{~d} \mathbf{r}_{3} \ldots \mathrm{d} \mathbf{r}_{N} \\
&=\quad \Xi_{Q P}^{k l} \psi_{k}\left(\mathbf{r}_{1}\right) \psi_{l}\left(\mathbf{r}_{1}\right)
\end{aligned}
$$

where in the second line we have used the expansion of a determinant along its first row, $M_{j}^{Q}\left(\mathbf{r}_{2}, \ldots, \mathbf{r}_{N}\right)$ is the minor of $\psi_{j}\left(\mathbf{r}_{1}\right)$ in $\Phi_{Q}\left(\left(\mathbf{r}_{(N)}\right)\right.$, and the matrix $\underline{s}$ is defined as the square root of a matrix,

$$
\underline{s}=\underline{S}^{-\frac{1}{2}},
$$

whose elements are given by the integrals,

$$
S_{i j}=\int \frac{n(\mathbf{r}) \phi_{i}(\mathbf{r}) \phi_{j}(\mathbf{r})}{\left[C^{P} C^{Q} \Xi_{P Q}^{k l} s_{k}^{u} s_{l}^{u} \phi_{u}(\mathbf{r}) \phi_{u}(\mathbf{r})\right]} \mathrm{d} \mathbf{r} .
$$

The non-linear nature of the transformation, $\underline{S}$, between the set of functions, $\left\{\phi_{j}(\mathbf{r})\right\}$ and $\left\{\psi_{k}\right\}$, is clear. The existence and convergence of $\underline{S}$ has been demonstrated by Cioslowski $[19,20]$.

Cioslowski [18] shows that the set of orbitals that form the Slater determinant, $\Phi_{0}\left(\mathbf{r}_{(N)}\right)$ take the form,

$$
\psi_{j}(\mathbf{r})=\frac{\sqrt{n(\mathbf{r})} s_{j}^{u} \phi_{u}(\mathbf{r})}{\sqrt{\left\langle\phi(\mathbf{r})\left|\underline{S}^{-1}\right| \phi(\mathbf{r})\right\rangle}},
$$

where,

$$
S_{i j}=\int \frac{n(\mathbf{r}) \phi_{i}(\mathbf{r}) \phi_{j}(\mathbf{r})}{\left[\left\langle\phi(\mathbf{r})\left|\underline{S}^{-1}\right| \phi(\mathbf{r})\right\rangle\right]} \mathrm{d} \mathbf{r} .
$$

In the last two expressions, a vector-matrix notation is used, so that $\left\langle\phi(\mathbf{r})\left|\underline{S}^{-1}\right| \phi(\mathbf{r})\right\rangle=\sum_{k l} \phi_{k}(\mathbf{r})\left[\underline{S}^{-1}\right]_{k l} \phi_{l}(\mathbf{r})$.

We now examine the properties of the orbitals defining $\Phi_{0}\left(\mathbf{r}_{(N)}\right)$.

Clearly, the set, $\left\{\phi_{j}(\mathbf{r})\right\}$, is arbitrary and hence not a functional of the density. Because of this, the matrix, $\underline{S}$, whose value depends on $\left\{\phi_{j}(\mathbf{r})\right\}$ is not a functional of the density either, and consequently neither are the orbitals, $\psi_{j}(\mathbf{r})$. However, the set of orbitals forming $\Phi_{0}(\mathbf{r})$ is uniquely defined regardless of the choice of $\left\{\phi_{j}(\mathbf{r})\right\}$.

Furthermore, because of the explicit presence of the density in Eq. (A.8), the matrix, $\underline{S}$, possesses a parametric derivative with respect to the density,

$$
\begin{gathered}
\frac{\delta \underline{S}}{\delta n\left(\mathbf{r}^{\prime}\right)}=\frac{\left|\phi\left(\mathbf{r}^{\prime}\right)\right\rangle\left\langle\phi\left(\mathbf{r}^{\prime}\right)\right|}{\left\langle\phi\left(\mathbf{r}^{\prime}\right)\left|\underline{S}^{-1}\right| \phi\left(\mathbf{r}^{\prime}\right)\right\rangle} \\
+\quad \int \frac{n(\mathbf{r})|\phi(\mathbf{r})\rangle\langle\phi(\mathbf{r} \mid)}{\left[\left\langle\phi(\mathbf{r})\left|\underline{S}^{-1}\right| \phi(\mathbf{r})\right]^{2}\right.} \\
\times \quad \operatorname{Tr}\left[\underline{S}^{-1}|\phi(\mathbf{r})\rangle\langle\phi(\mathbf{r})| \underline{S}^{-1} \frac{\delta \underline{S}}{\delta n\left(\mathbf{r}^{\prime}\right)}\right] \mathrm{d} \mathbf{r},
\end{gathered}
$$


an equation to be solved for the derivatives, $\frac{\delta S_{i j}}{\delta n(\mathbf{r})}($ see $[19,20])$. (For a fixed set $\left\{\phi_{j}(\mathbf{r})\right\}$, one can view $S_{i j}$ as a functional of the density, and refer to the parametric derivative as a functional derivative. At the same time, the derivative with respect to the density, $n(\mathbf{r})$, requires no knowledge of $\underline{S}$ for any density other than $n(\mathbf{r})$ ). This defines the parametric derivative of the basis functions, $\psi_{j}(\mathbf{r})$, with respect to the density for each choice of auxiliary functions, evaluated at the particular density. Consequently, it defines a unique derivative of any wave function leading to the density that is independent of the choice of auxiliary functions.

To summarize: Each density, $n(\mathbf{r})$, defines its own space of parametric (functional) differentiation of individual orbitals used to construct the antisymmetric, $N$-particle wave functions, $\Psi\left(\mathbf{r}_{(N)} ;[n]\right)$, that lead to a density. Thus, all orbitals and corresponding wave functions that lead to a density possess uniquely defined parametric derivatives with respect to the density.

\section{Appendix B. Equidensity Basis}

Although Cioslowski's procedure for taking derivatives is formally exact, it is computationally exceedingly difficult to implement. A computationally viable procedure can be constructed based on the existence of the derivative, and the properties of expansions in a complete and orthonormal basis: Namely, all properties of a function, derivatives included, are preserved in such an expansion.

In this case, each orbital is expanded in terms of an orthonormal and complete basis that is explicitly written in terms of the density, allowing differentiation with respect to the density. The basis in question, referred to here as the equidensity basis is that discussed in detail in reference [5] (see also references to previous work there). For the one-dimentional case considered here, these elements of the basis also known as Harriman orbitals, see references in [5] take the form,

$$
\psi_{k}(x)=\sqrt{\frac{n(x)}{N}} \exp \left\{-\mathrm{i} k \int_{-\infty}^{x} n\left(x^{\prime}\right) \mathrm{d} x^{\prime}\right\},
$$

an expression that can be immediately differentiated functionally with respect to the density. It follows that any one of the orbitals that sum up to a density can be expanded in terms of the eqidensity basis,

$$
\phi_{j}(x)=\sum_{k} a_{k}^{j} \psi_{k}(x),
$$

where

$$
a_{k}^{j}=\int \phi_{j}(x) \psi_{k}^{*}(x) \mathrm{d} x,
$$

is an overlap integral. The immediate differentiation of the orbitals in terms of differentiation of the equidensity basis is clear.

[1] P. Hohenberg and W. Kohn. Inhomogeneous electron gas. Phys. Rev., 136(3B):B864-B871, Nov 1964.

[2] R. G. Parr and C. Y. Yang. Density Functional Theory of Atoms and Molecules. Oxford University Press, Oxford, 1989.

[3] R. M. Dreitzler and E. K. U. Gross. Density Functional Theory. Springer Verlag, Berlin, New York, 1990.
[4] Weitao Yang, Paul W. Ayers, and Qin Wu. Potential functionals: dual to density functionals and solution to the v-representability problem. Phys. Rev. Lett., 92(14):146404-1-146404-4, 2004.

[5] Gil Zumbach and Klaus Maschke. New approach to the calculation of density functionals. Phys. Rev. A, 28(2):544-554, Aug 1983.

[6] Mel Levy. Electron densities in search of hamiltonians. Phys. Rev. A, 26:1200-1208, Sep 1982.

[7] Elliot H. Lieb. Density Functionals for Coulomb Systems. MIT Press Cambridge, Massachusetts, 1982. See also, E. H. Lieb, in Density Functional Methods in Physics, ed. R. M. Dreizler, NATO ASI Series B123, (Plenum, New York, 1985) p.31; and E. H. Lieb, in Int. J. Quantum Chem. 24, 243 (1983).

[8] H. English and R. English. Hohenberg-kohn theorem and non-vrepresentable densities. Physica, 121 A:253-268, 1983.

[9] H. Englisch and R. Englisch. Exact density functionals for ground-state energies. i. general results. physica status solidi (b), 123(2):711-721, 1984.

[10] H. Englisch and R. Englisch. Exact density functionals for ground-state energies ii. details and remarks. physica status solidi (b), 124(1):373-379, 1984.

[11] Eberhard Engel. Orbital-Dependent Functionals for the ExchangeCorrelation Energy: A Third Generation of Density Functionals, in: A Primer in Density Functional Theory, volume 620 of Lecture Notes in Physics, chapter 2, pages 56-122. Springer, Berlin, Heidelberg, New York, 2003. ISBN 978-3-540-03083-6.

[12] T. Grabo, T. Kreibich, S. Kurth, and E. Gross. Strong Coulomb Correlations in Electronic Structure: Beyond the Local Density Approximation, pages 203-311. Gordon and Breach Science Publishers, Amsterdam, 2000.

[13] W. Yang and Q. Wu. Phys. Rev. Lett., 89:143002, 2002.

[14] Stephan Kümmel and John P. Perdew. Simple iterative construction of the optimized effective potential for orbital functionals, including exact exchange. Phys. Rev. Lett., 90(4):043004, Jan 2003.

[15] Stephan Kümmel and Leeor Kronik. Orbital-dependent density functionals: Theory and applications. Rev. Mod. Phys., 80(1):3-60, Jan 2008.

[16] Mel Levy. Universal variational functionals of electron densities, firstorder density matrices, and natural spin-orbitals and solution of the vrepresentability problem. Proceedings of the National Academy of Sciences, 76(12):6062-6065, 1979.

[17] A. Gonis. functionals and functional derivatives of wave functions and densities. W. Journal Condens. Matt. Phys., 4:179-199, 2014.

[18] J. Cioslowski. Density functionals for the energy of electronic systems: Explicit variational construction. Phys. Rev. Lett., 60:2141-2143, May 1988.

[19] J. Cioslowski. Density driven self-consistent field method. i. derivation and basic properties. The Journal of Chemical Physics, 89(8):48714874, 1988

[20] J. Cioslowski. Density driven self-consistent field method. ii. construction of all one-particle wave functions that are orthonormal and sum up to a given density. International Journal of Quantum Chemistry, Quantum Chemistry Symposium 23(8):255-262, 1989.

[21] J. T. Chayes, L. Chayes, and M. B. Ruskai. Density functional approach to quantum lattice systems. J. Statist. Phys., 38:493, 1985.

[22] R. P. Feynman. Forces in Molecules. Physical Review, 56:340-343, August 1939. 